\title{
The Combination of Vascular Endothelial Growth Factor and Stromal Cell-Derived Factor Induces Superior Angiogenic Sprouting by Outgrowth Endothelial Cells
}

\author{
Erin M. Anderson ${ }^{a, b}$ David J. Mooney ${ }^{a, b}$ \\ ${ }^{a}$ School of Engineering and Applied Sciences and bWyss Institute for Biologically Inspired Engineering, \\ Harvard University, Cambridge, Mass., USA
}

\section{Key Words}

Angiogenesis - Endothelial progenitor cells - Therapeutic neovascularization - Vascular endothelial growth factor .

Stromal cell-derived factor

Abstract

Endothelial progenitor cells are being broadly explored for the treatment of ischemic cardiovascular diseases, but their response to molecules commonly used to promote the growth of new blood vessels has not been fully characterized. In this study, angiogenic sprout formation in a 3-dimensional, in vitro model by one type of endothelial progenitor, outgrowth endothelial cells (OECs), was characterized in response to exposure to stromal cell-derived factor (SDF) and vascular endothelial growth factor (VEGF) and then compared to mature endothelial cells. Exposure to SDF alone did not increase angiogenic sprouting in comparison to control media, while a combination of VEGF and SDF demonstrated greater potency than VEGF alone for all cell types. Together, VEGF and SDF reduced the sprout initiation time and maintained sprouting levels over time. In direct competition with mature endothelial cells, OECs preferentially localized to the tip cell position, suggesting an enhanced sprouting potential. Overall, these results reveal the impact of the combina- tion of VEGF and SDF on endothelial cell sprouting, and support the enhanced potential of OECs, as opposed to mature endothelial cells, for treating ischemic diseases.

(c) 2015 S. Karger AG, Basel

\section{Introduction}

Ischemic cardiovascular diseases affect over 25 million Americans [1], supporting the need for new therapeutic strategies to reduce disease prevalence. The discovery of endothelial progenitor cells has led to significant efforts to use these cells instead of mature endothelial cells for the treatment of ischemic tissue [2-5]. Specifically, late-outgrowth endothelial cells (OECs), also called endothelial colony-forming cells, which are isolated from cord blood, have been widely explored as a therapeutic agent, due to their high proliferation rate and enhanced vasculogenic potential in implanted 3-dimensional matrices when compared to similar cells isolated from adult blood or mature endothelial cells $[6,7]$. However, the responsiveness of OECs to growth factors commonly used for endothelial progenitor cell recruitment and therapeutic angiogenesis has not been explored.

\section{KARGER 125}

(c) 2015 S. Karger AG, Base

$1018-1172 / 15 / 0521-0062 \$ 39.50 / 0$

E-Mail karger@karger.com

www.karger.com/jvr
David J. Mooney

School of Engineering and Applied Sciences, Harvard University

319 Pierce Hall, 29 Oxford Street

Cambridge, MA 02138 (USA)

E-Mail mooneyd@seas.harvard.edu 
We examined the impact of exposure to vascular endothelial growth factor (VEGF) and stromal cell-derived factor (SDF), individually and in combination, on OEC and mature endothelial cell sprouting. We utilized a physiologically relevant, 3-dimensional assay in which endothelial cell-seeded beads are suspended in a fibrin matrix and exposure to angiogenic factors encourages sprouting into the fibrin [8]. This sprouting assay replicates the initial stages of angiogenesis, including the loosening of interendothelial contacts, followed by endothelial cell migration and the formation of sprouts, similar to the in vivo angiogenic process [9]. The extent of sprouting measured in this assay has been found to be predictive of the in vivo response to angiogenic factors [10-12].

VEGF and SDF were chosen for these studies because of their roles in neovascularization and in the recruitment of circulating endothelial progenitor cells. VEGF is a wellknown angiogenic factor, and may also play a role in endothelial progenitor recruitment $[9,13,14]$. The effect of VEGF concentration and spatial and temporal gradients on the sprouting of mature endothelial cells has previously been explored $[10,11]$. SDF is a cytokine that plays a role in many steps of the cell-trafficking process, is a known chemoattractant for endothelial progenitors and is also claimed to be angiogenic, as it helps to restore perfusion to ischemic tissue when delivered locally [15-19]. However, it is not clear if SDF plays a direct role in angiogenesis, because so far its role has been indirect, i.e. by recruiting various stem and progenitor cells to sites of new vessel growth [19-24]. While SDF may assist with endothelial progenitor targeting, its direct effect on the neovascularization potential of OECs or mature endothelial cells has not been demonstrated. Here, we directly compared stimulation by SDF and VEGF, individually and in combination, in order to gain insight into the potential role of $\mathrm{SDF}$ in promoting OEC sprouting angiogenesis.

Qualitative interactions between mature endothelial cells and OECs have been previously reported [25], but here we quantitatively compared the sprout-forming potential of OECs with 2 types of mature endothelial cells, human umbilical vein endothelial cells (HUVECs) and human microvascular endothelial cells (HMVECs), by seeding both cell types on the beads together. Overall, the results of these studies elucidate the effect of SDF on endothelial sprouting angiogenesis in vitro and demonstrate the enhanced sprouting of OECs in comparison to that by mature endothelial cells with VEGF and SDF stimulation. The findings presented here increase our understanding of OEC behavior, and may subsequently lead to improved, more effective clinical uses of OECs.

\section{Materials and Methods}

\section{Cell Culture and Characterization}

Human cord blood was used for OEC isolation. The mononuclear cell fraction from cord blood was collected after density segregation with Histopaque 1077 (Sigma No. 10771-500 ml). Remaining red blood cells were lysed and cells were washed with Hanks' balanced salt solution. OECs were plated in EGM-2MV (Lonza No. CC-3202) supplemented with 10\% FBS onto collagencoated plates (BD No. 354400). Medium was changed after 2 days, and then every day until colonies appeared (on days 10-14). Approximately a half to two thirds of the wells contained at least one colony. Cells were replated and maintained in EGM-2MV medium while in use; OECs were used between passages 2 and 6. HMVECs (Lonza No. CC-2543) and HUVECs (Lonza No. C-2517A) were cultured in EGM-2MV and EGM-2 (Lonza No. CC-3162), respectively, and were used between passages 3 and 6. CD31, VEGFR2 and CXCR4 receptor expression on OECs, HMVECs and HUVECs was characterized by flow cytometry using fluorescently labeled antibodies (Biolegend Nos. 303109, 359903 and 306505, respectively). Proliferation of each cell type was compared using an XTT-based assay kit (ATCC No. 30-1011K) according to the manufacturer's instructions. Briefly, an equal number of OECs, HMVECs and HUVECs were seeded into 48-well plates, and proliferation was measured at $0,24,48$ and $72 \mathrm{~h}(\mathrm{n}=4)$.

\section{Sprouting Assays}

Sprouting assays were performed similarly to those previously described $[8,10,11]$. Here, HMVECs, HUVECs or cord bloodisolated OECs were seeded onto collagen-coated beads $(200 \mu \mathrm{m}$ in diameter; Cytodex ${ }^{\mathrm{TM}} 3$ Microcarriers, GE Healthcare No. 17-048501 ) by alternating between rest and spinning states for $4 \mathrm{~h}$. In some conditions, OECs were stained with octadecylrhodamine B chloride dye (Life Technologies; No. O-246) and then seeded onto beads in a 1:1 ratio with unstained HMVECs or HUVECs. The cell-seeded beads were cultured in an orbital shaker until the cells became confluent. For conditions with rhodamine-stained OECs and unstained HUVECs or HMVECs coseeded on the beads, the percentage of each cell type on the beads was measured by flow cytometry prior to beginning the assays (BD LSRFortessa, Franklin Lakes, N.J., USA). To initiate sprouting assays, $1 \mathrm{ml}$ of the bead suspension was mixed with $3 \mathrm{ml}$ of $2 \mathrm{mg} / \mathrm{ml}$ fibrinogen (Millipore; No. EMD 341576) and $400 \mu \mathrm{l}$ of $500 \mu \mathrm{g} / \mathrm{ml}$ aprotinin (Sigma; No. A4529-5MG), and then $250 \mu \mathrm{l}$ of this solution was mixed with $200 \mu \mathrm{l}$ of $2 \mathrm{U} / \mathrm{ml}$ thrombin (Sigma; No. F3879-250MG) in a 24-well plate to form a fibrin gel. Each well contained 100-150 beads, which occupied approximately $0.1 \%$ of the total gel volume. After the gels had solidified, $600 \mu \mathrm{l}$ of media was added to the top of the fibrin gel [EBM2 + 5\% FBS was used as the control medium, with rhSDF-1 $\alpha=100 \mathrm{ng} / \mathrm{ml}$ (R\&D Systems; No. 350-NS), rhVEGFA-165 = $50 \mathrm{ng} / \mathrm{ml}$ (R\&D Systems; No. 293-VE) or the combination added as control conditions]. Media were changed every $24 \mathrm{~h}$ for 3 days. Increasing $(10,100$ and $190 \mathrm{ng} / \mathrm{ml})$, stable (100, 100 and $100 \mathrm{ng} / \mathrm{ml})$ and decreasing $(190,100$ and $10 \mathrm{ng} / \mathrm{ml})$ profiles of SDF on days 1, 2 and 3, respectively, were also overlaid on top of a decreasing $(95,50$ and $5 \mathrm{ng} / \mathrm{ml})$ VEGF profile, in order to determine the effects that temporally controlled cytokine presentation has on sprouting. At 24, 48 and $72 \mathrm{~h}$, the assays were stopped by rinsing in PBS and fixing in $4 \%$ paraformaldehyde. The number of sprouts per bead was counted on a Zeiss Axio Observer Z1 microscope 


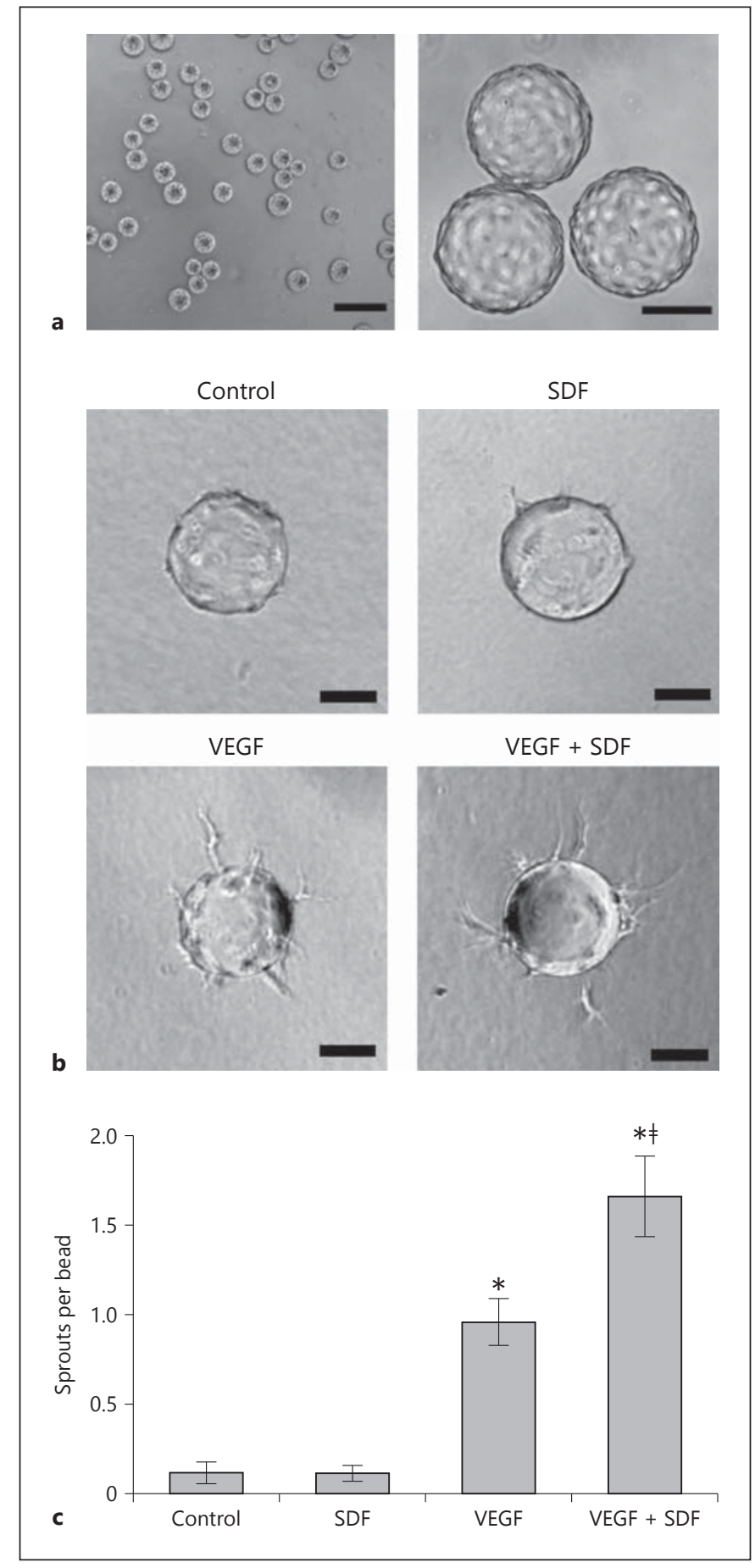

Fig. 1. OEC adherence to beads and sprout formation in response to SDF and VEGF alone and in combination at $72 \mathrm{~h}$. a A confluent layer of OECs was adhered to beads prior to seeding into the fibrin gel at the start of the experiment. Scale bar: $500 \mu \mathrm{m}$ (left), $100 \mu \mathrm{m}$ (right). b Images of OEC sprouts formed after $72 \mathrm{~h}$ of exposure to control media, or media supplemented with $100 \mathrm{ng} / \mathrm{ml} \mathrm{SDF,} 50 \mathrm{ng} / \mathrm{ml} \mathrm{VEGF}$ or the combination. Scale bar: $100 \mu \mathrm{m}$. c Quantification of the number of sprouts per bead formed by OECs (mean \pm SD; $\mathrm{n}=4)$. ${ }^{*} \mathrm{p}<0.05 \mathrm{com}$ pared to control condition, ${ }^{\dagger} \mathrm{p}<0.05$ compared to VEGF condition.
(Zeiss, Thornwood, N.Y., USA). A sprout was defined as more than 1 cell protruding from the bead while remaining connected to the bead surface, as previously described $[8,10]$. Approximately 100 beads per well were counted, with 4 wells per condition.

\section{Time-Lapse Imaging of OEC Sprouting}

Sprouting assays were prepared as described above. A plate of OEC-seeded beads in fibrin gel was placed in a live-cell imaging chamber on a Zeiss Axio Observer Z1 microscope, prewarmed to $37^{\circ} \mathrm{C}$ and set to $5 \% \mathrm{CO}_{2}$. Metamorph Imaging Software (Molecular Devices, Sunnyvale, Calif., USA) was used for time-lapse image capture: $200-\mu \mathrm{m}$ stacks (10- $\mu \mathrm{m}$ steps) of phase-contrast images over 10 fields of view, each containing 1 cell-seeded bead, were imaged per well, with 4 wells imaged per condition. Image stacks were captured every $20 \mathrm{~min}$ for $24 \mathrm{~h}$. For each bead, the time at which the first OEC protruded from the bead was recorded as the sprout initiation time.

\section{Statistical Analysis}

Data were compared by means of the unpaired Student $t$ test (two-tailed), with $\mathrm{p}<0.05$ considered significant.

\section{Results}

OECs readily adhered to the beads and proliferated until they formed a confluent layer of cells (fig. 1a), similar to previously reported bead-coating by HMVECs and HUVECs $[10,11,26]$. Sprouting assays were used to determine the effects of VEGF and SDF on OEC sprouting. OEC-covered beads were placed in a fibrin matrix, and the cells migrated from the beads to form sprouts, mimicking the initial stages of angiogenesis. After 3 days, SDF did not support sprout formation whereas VEGF, a known angiogenic cytokine, induced sprout formation, and, surprisingly, the combination of VEGF and SDF promoted the most sprout formation (fig. 1b, c). In addition to the positive effect of a constant presence of SDF with VEGF, the temporal presentation of SDF may be important for sprouting. This effect is also observed with VEGF alone, where a decreasing concentration of VEGF over time is known to stimulate the best sprout formation by HMVECs [11]. The effect of increasing, decreasing or stable profiles of SDF, overlaid on stable and decreasing profiles of VEGF, was tested at the day 3 time point (online suppl. fig. 1a; for all online suppl. material, see www.karger.com/doi/10.1159/000382129). All profiles of SDF presentation, in combination with stable and decreasing VEGF profiles, supported more sprout formation than VEGF alone (online suppl. fig. 1b).

The positive effect of VEGF and SDF on endothelial sprouting may be achieved by promoting a faster and more continuous sprout formation or by maintaining the 


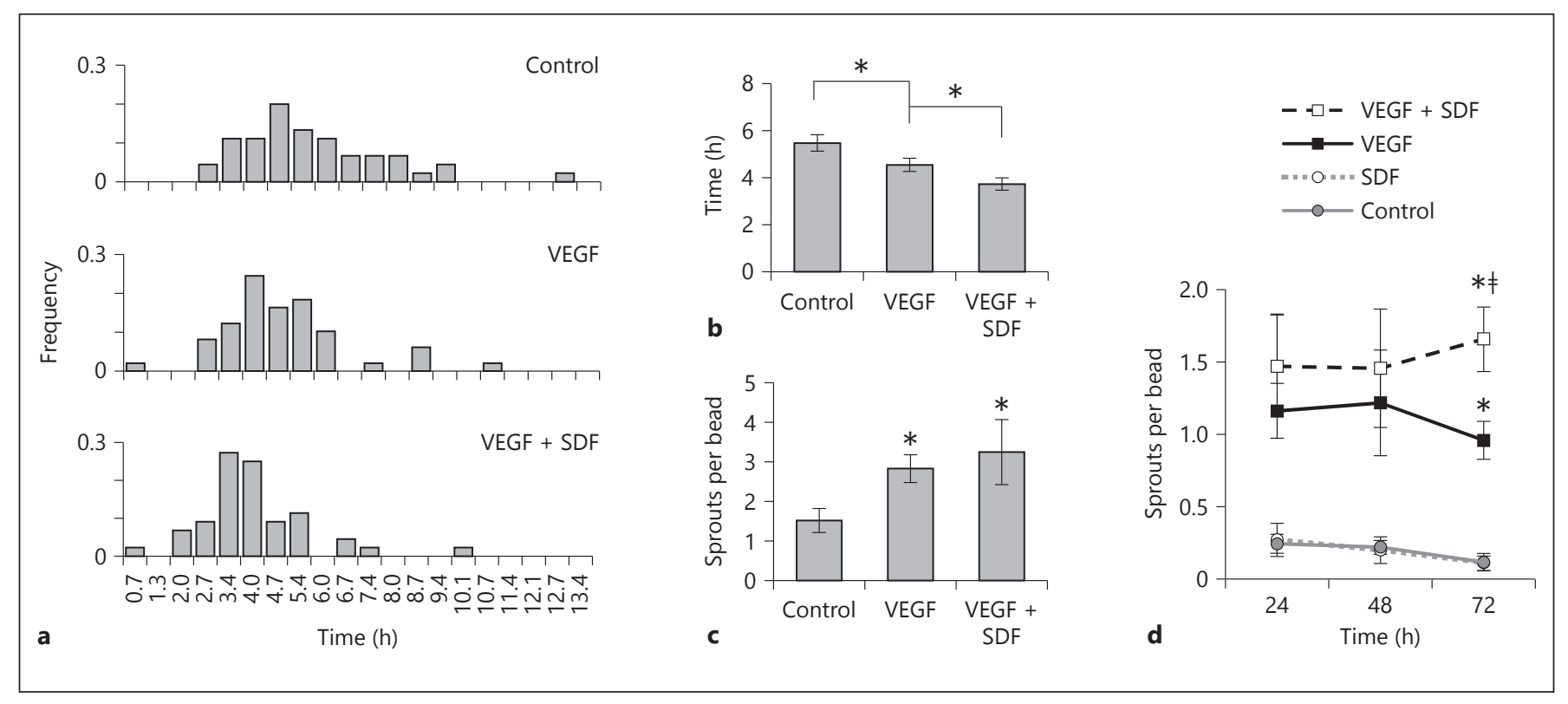

Fig. 2. Kinetics of OEC sprout formation and maintenance in response to VEGF and SDF. a Histograms of sprout initiation time for OECs with control media or media supplemented with VEGF or VEGF + SDF. b Population mean sprout initiation times $[$ mean \pm SEM; control $(n=45)$, VEGF $(n=49)$, VEGF + SDF

sprouts for longer. To analyze the kinetics of initial sprout formation, time-lapse microscopy was used to image the OECs in this sprouting assay (online suppl. fig. 2; online suppl. videos). The time at which the first cell protruded from the bead was recorded as the sprout initiation time (fig. 2a). The condition of SDF alone was not tested here because previously, it had not led to an increase in sprouting over the control. Both the VEGF condition and VEGF and SDF in combination showed faster sprout initiation times relative to the control, with VEGF alone reducing the initiation time by $1 \mathrm{~h}$ and the combination of VEGF and SDF reducing it further (by $50 \mathrm{~min}$; fig. 2a, b). The total effect of reduced initiation time on sprouting could be observed at $8 \mathrm{~h}$, where both conditions with VEGF already showed significantly more sprouts per bead relative to the control (fig. 2c). To investigate the effect of VEGF and SDF over time, the number of sprouts per bead was compared after 24, 48 and $72 \mathrm{~h}$ (fig. 2d). At $24 \mathrm{~h}$, more sprouts formed when VEGF was present, compared to the control and to SDF alone, a possible continued effect of the reduced sprout initiation time. While the VEGF condition and VEGF and SDF in combination displayed similar levels of sprouting at 24 and $48 \mathrm{~h}$, significant differences were observed at $72 \mathrm{~h}$ as well as at later points in time [27]. Together, these data demonstrate that the com-
( $\mathrm{n}=44)$ ]. c Quantification of the number of sprouts per bead formed after $8 \mathrm{~h}$ (mean $\pm \mathrm{SD} ; \mathrm{n}=4)$. d Quantification of the number of sprouts formed by OECs at 24, 48 and $72 \mathrm{~h}$ (mean $\pm \mathrm{SD} ; \mathrm{n}=$ 4). ${ }^{*} \mathrm{p}<0.05$ compared to all other conditions, ${ }^{\ddagger} \mathrm{p}<0.05$ compared to VEGF condition.

bination of VEGF and SDF potently promotes sprouting, possibly by multiple mechanisms, including a reduced sprout initiation time, the enhanced maintenance of the sprouts that have formed and/or by encouraging continuous sprouting over time.

Next, we observed the effect of VEGF and SDF on mature endothelial sprout formation, and then compared it to the sprouting potential of OECs. After $72 \mathrm{~h}$, HMVECs and HUVECs both demonstrated responses to the growth factors similar to those with OECs, with the VEGF and SDF combination yielding the most sprouts per bead (fig. 3a, b). Since the absolute number of sprouts per bead can vary significantly from experiment to experiment, the data for each cell type were normalized so as to compare sprout maintenance in response to growth factor treatment over time. OECs tended to maintain sprouts better than HMVECS and HUVECs across all conditions, with significant differences arising for some comparisons (fig. 3c). In order to rigorously compare the sprout-forming potential of endothelial progenitors and mature endothelial cells, OECs and HMVECs or OECs and HUVECs were placed in direct competition by seeding both cell types onto the beads together (fig. 4a). OECs were often observed in the tip cell position in sprouts, and even sprouts which had a HMVEC as the tip cell were often 


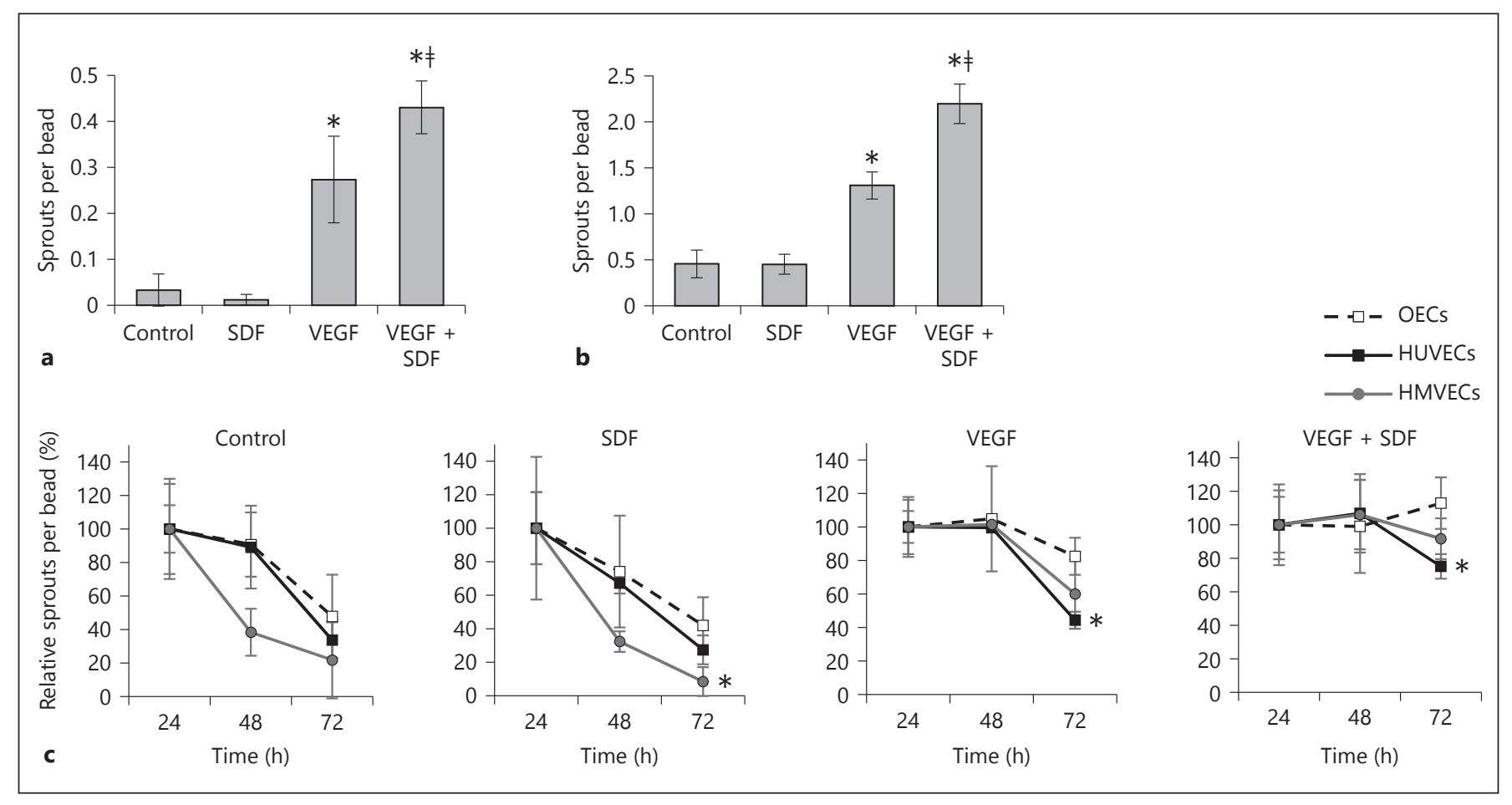

Fig. 3. HMVEC and HUVEC response to VEGF and SDF in comparison to OECs. Quantification of the number of sprouts per bead formed by HMVECs (a) and HUVECs (b) after $72 \mathrm{~h}$ (mean \pm SD; $\mathrm{n}=4) .{ }^{*} \mathrm{p}<0.05$ compared to control condition, ${ }^{\dagger} \mathrm{p}<0.05$ com-

supported at the stalk by an OEC (fig. 4b). Many sprouts were formed completely by OECs (fig. 4c). The percentage of sprouts with OECs in the tip cell was subsequently quantified. Compared to HMVECs, even though initially only $38 \%$ of the cells on the beads were OECs (online suppl. fig. 3), at 24,48 and $72 \mathrm{~h}, 60-70 \%$ of the sprouts had an OEC as the tip cell across all conditions (fig. 4d). Similar trends were revealed when OECs and HUVECs were seeded onto the beads together (fig. 4e). When OECs and HMVECs or HUVECs were cultured together, the combination of VEGF and SDF still promoted the most sprout formation, followed by VEGF alone, while the control condition and SDF alone showed significantly fewer sprouts (fig. 4f, g). Furthermore, the increased sprout maintenance and localization to the tip cell position for OECs compared to mature endothelial cells were not as a result of enhanced OEC proliferation or due to significant differences in VEGFR2, CXCR4 or CD31 receptor expression (online suppl. fig. 4, 5). Direct comparison of OECs to mature endothelial cell types in these assays therefore demonstrates the greater sprouting potential of OECs. pared to VEGF condition. c Comparison of sprout maintenance between cell types in various media conditions. Data were normalized to the number of sprouts per bead at $24 \mathrm{~h}($ mean $\pm S D ; n=4)$. $* \mathrm{p}<0.05$ compared to OECs.

\section{Discussion}

These studies revealed the potency of VEGF and SDF in promoting angiogenic sprouting in vitro, and compared the sprouting ability of OECs and mature endothelial cells. Exposure to SDF alone did not increase the number of sprouts per bead in comparison to control media for any cell type analyzed, while the combination of VEGF and SDF significantly enhanced sprouting to above the levels achieved with VEGF, a known angiogenic factor. The combination of VEGF and SDF also reduced the sprout initiation time and resulted in a higher number of sprouts per bead at $72 \mathrm{~h}$ compared to VEGF alone for OECs, HMVECs and HUVECs. In direct comparison to mature endothelial cells, OECs maintained sprouts better and preferentially localized to the tip cell position, which suggests they may exhibit a greater neovascularization potential than HMVECs or HUVECs.

The combination of VEGF and SDF had a significant effect on sprouting by OECs and endothelial cells. First, VEGF and SDF together increased sprouting at $72 \mathrm{~h}$ for OECs, HUVECs and HMVECs compared to VEGF expo- 


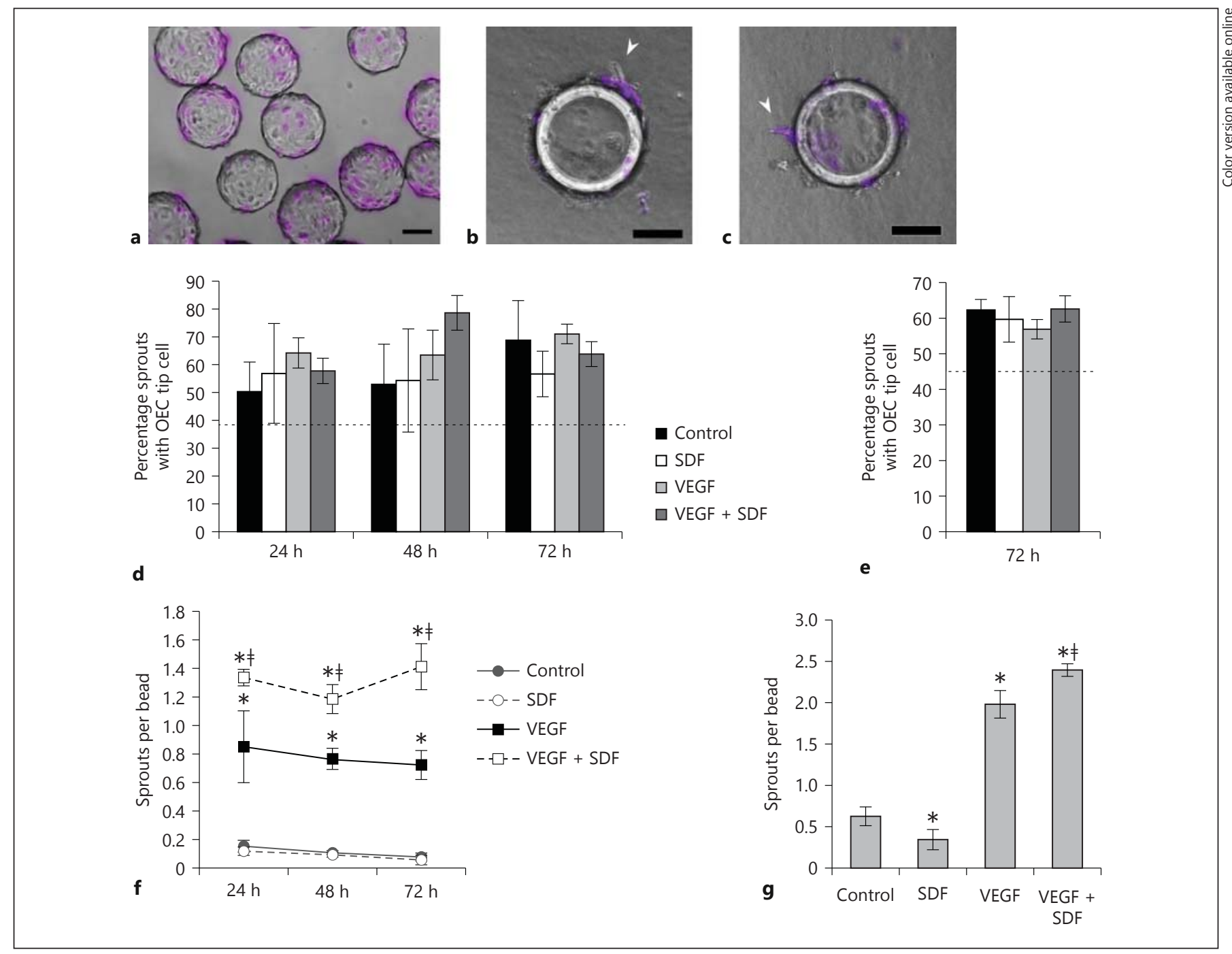

Fig. 4. Direct comparison of the sprouting potential of OECs, HMVECs and HUVECs. a OECs and HMVECs were seeded onto $200-\mu \mathrm{m}$ beads. OECs are shown in magenta (please see online version for color figure). Scale bar: $100 \mu \mathrm{m}$. b Sprout formed by a HMVEC and supported by an OEC at the base (arrowhead). Scale bar: $100 \mu \mathrm{m}$. c Sprout formed by OECs (arrowhead). Scale bar: 100 $\mu \mathrm{m}$. The percentage of sprouts with an OEC as the tip cell when

sure alone, SDF alone or control media. VEGF is known to promote the formation of blood vessels in vitro and in vivo, and has previously been tested in this assay $[10,11]$. It has been suggested that SDF is angiogenic because it supports tube formation in 2-dimensional assays [21], even though these are not rigorous assays of angiogenic capability. Here, SDF alone did not stimulate sprout formation at any point in time, but it did enhance the impact of VEGF on sprouting. In previous studies [22, 28], the observation of an improvement in tissue perfusion following the local delivery seeded with HMVECs (d) or HUVECs (e) is shown; the dashed line indicates the initial percentage of cells that were OECs prior to seeding in fibrin. $\mathbf{f}$ Quantification of total sprouts per bead formed by a combination of OECs and HMVECs at 24,48 and $72 \mathrm{~h}$. g Total number of sprouts per bead formed by a combination of OECs and HUVECs at $72 \mathrm{~h}($ mean $\pm S D ; n=4) .{ }^{*} \mathrm{p}<0.05$ compared to control condition, ${ }^{\ddagger} \mathrm{p}<0.05$ compared to VEGF condition. 
Observations of sprouting kinetics suggest that the increased sprouting at early time points in response to VEGF and SDF may reflect the reduced sprout initiation time, while the differences at later time points may result from more continuous sprout formation and/or by maintaining sprouts for longer. The initiation time of only the first sprout formed was recorded here, but the initiation time, total time of sprout maintenance and retraction time for all sprouts between 0 and $72 \mathrm{~h}$ was not determined. In addition, VEGF and SDF may act synergistically to enhance the overall migratory ability of OECs, which could help promote the continuous formation of sprouts by individual cells, since sprouting is initiated by the migration of a cell into the fibrin matrix. Therefore, even if the retraction rate for all of the conditions was similar, an increase in the total number of sprouts formed could still be observed. Analysis of the full dynamics would reveal which mechanism is responsible for the greater number of sprouts per bead found at the later time points. In any case, these experiments suggest that the combination of VEGF and SDF has strong potential to support the formation of new blood vessels by OECs that have localized to ischemic tissue.

The 3-dimensional sprouting assay was also used to compare the response of OECs to that of HMVECs or HUVECs to VEGF and SDF as well as their ability to form sprouts by coseeding both cell types onto the beads, and allowing them to directly compete for tip cell position. OECs demonstrated improved sprout maintenance, and were preferentially found at the tip cell location compared to HMVECs and HUVECs. Preferential sprout formation by OECs was not due to higher CXCR4 or VEGFR2 receptor expression and was also not a result of an enhanced proliferation rate in response to VEGF or VEGF and SDF. Enhanced proliferation by OECs would also be expected to lead to an increasing fraction of OEC sprouts over time, which was not observed. These experiments demonstrated that OECs have an increased capability to form and maintain sprouts, and also to function in concert with mature endothelial cells. These results are further in agreement with other models of vessel network formation, in which evenly distributed OECs in a 3-dimensional collagen gel matrix condensed to form vessellike structures with an increased microvessel density compared to networks of HMVECs [6]. Therapeutically, this suggests that if OECs are correctly located at sites of new blood vessel growth, they should exhibit a higher angiogenic potential than the resident endothelial cells, but this was not examined here.

Overall, we have shown that SDF does not directly promote angiogenic sprouting by OECs or mature endothelial cells, but that the combination of VEGF and SDF can dramatically increase sprouting, likely by reducing the sprout initiation time and improving sprout maintenance. This combination of factors may potentially increase microvessel density in tissue-engineered vascular networks as well as in models of cancer or ischemic disease [31, 32]. The results of these experiments also emphasize the enhanced sprouting capacity of OECs compared to that of mature endothelial cells, and support further investigation of the clinical uses of these cells.

\section{Acknowledgments}

The authors would like to acknowledge Thomas Ferrante at the Wyss Institute for his help in imaging the sprouting assays. This research was supported by the National Institutes of Health (RO1 HL069957) and the Wyss Institute for Biologically Inspired Engineering at Harvard University.

\section{References}

1 Lloyd-Jones D, Adams RJ, Brown TM, Carnethon M, Dai S, De Simone G, et al: Heart disease and stroke statistics - 2010 update: a report from the American Heart Association. Circulation 2010;121:e46-e215.

2 Asahara T, Murohara T, Sullivan A, Silver M, van der Zee R, Li T, et al: Isolation of putative progenitor endothelial cells for angiogenesis. Science 1997;275:964-966.

3 Kawamoto A, Asahara T: Role of progenitor endothelial cells in cardiovascular disease and upcoming therapies. Catheter Cardiovasc Interv Off J Soc Card Angiogr Interv 2007;70: 477-484.
4 Kawamoto A, Losordo DW: Endothelial progenitor cells for cardiovascular regeneration. Trends Cardiovasc Med 2008;18:33-37.

$\checkmark 5$ Losordo DW, Dimmeler S: Therapeutic angiogenesis and vasculogenesis for ischemic disease: part II: cell-based therapies. Circulation 2004; 109:2692-2697.

-6 Melero-Martin JM, Khan ZA, Picard A, Wu $\mathrm{X}$, Paruchuri S, Bischoff J: In vivo vasculogenic potential of human blood-derived endothelial progenitor cells. Blood 2007;109:47614768.

7 Ingram DA, Mead LE, Tanaka H, Meade V, Fenoglio A, Mortell K, et al: Identification of a novel hierarchy of endothelial progenitor cells using human peripheral and umbilical cord blood. Blood 2004;104:2752-2760.

-8 Nehls V, Drenckhahn D: A novel, microcarrier-based in vitro assay for rapid and reliable quantification of three-dimensional cell migration and angiogenesis. Microvasc Res 1995; 50:311-322.

9 Carmeliet P: Mechanisms of angiogenesis and arteriogenesis. Nat Med 2000;6:389-395.

10 Chen RR, Silva EA, Yuen WW, Brock AA, Fischbach C, Lin AS, et al: Integrated approach to designing growth factor delivery systems. FASEB J 2007;21:3896-3903. 
11 Silva EA, Mooney DJ: Effects of VEGF temporal and spatial presentation on angiogenesis. Biomaterials 2010;31:1235-1241.

-12 Brudno Y, Ennett-Shepard AB, Chen RR, Aizenberg M, Mooney DJ: Enhancing microvascular formation and vessel maturation through temporal control over multiple proangiogenic and pro-maturation factors. Biomaterials 2013;34:9201-9209.

13 Asahara T, Takahashi T, Masuda H, Kalka C, Chen $\mathrm{D}$, Iwaguro $\mathrm{H}$, et al: VEGF contributes to postnatal neovascularization by mobilizing bone marrow-derived endothelial progenitor cells. EMBO J 1999;18:3964-3972.

14 Grunewald M, Avraham I, Dor Y, BacharLustig E, Itin A, Jung S, et al: VEGF-induced adult neovascularization: recruitment, retention, and role of accessory cells. Cell 2006;124: 175-189.

15 Petit I, Szyper-Kravitz M, Nagler A, Lahav M, Peled A, Habler L, et al: G-CSF induces stem cell mobilization by decreasing bone marrow SDF-1 and up-regulating CXCR4. Nat Immunol 2002;3:687-694.

16 Lévesque J-P, Hendy J, Takamatsu Y, Simmons PJ, Bendall LJ: Disruption of the CXCR4/CXCL12 chemotactic interaction during hematopoietic stem cell mobilization induced by GCSF or cyclophosphamide. J Clin Invest 2003;111:187-196.

17 Aiuti A, Webb IJ, Bleul C, Springer T, Gutierrez-Ramos JC: The chemokine SDF- 1 is a chemoattractant for human CD34+ hematopoietic progenitor cells and provides a new mechanism to explain the mobilization of CD34+ progenitors to peripheral blood. J Exp Med 1997;185:111-120.

18 De Falco E, Porcelli D, Torella AR, Straino S, Iachininoto MG, Orlandi A, et al: SDF-1 involvement in endothelial phenotype and isch- emia-induced recruitment of bone marrow progenitor cells. Blood 2004;104:3472-3482.

19 Yamaguchi J, Kusano KF, Masuo O, Kawamoto A, Silver M, Murasawa S, et al: Stromal cell-derived factor-1 effects on ex vivo expanded endothelial progenitor cell recruitment for ischemic neovascularization. Circulation 2003;107:1322-1328.

20 Kanda S, Mochizuki Y, Kanetake H: Stromal cell-derived factor-1alpha induces tube-like structure formation of endothelial cells through phosphoinositide 3-kinase. J Biol Chem 2003;278:257-262.

-21 Salvucci O, Yao L, Villalba S, Sajewicz A, Pittaluga S, Tosato G: Regulation of endothelial cell branching morphogenesis by endogenous chemokine stromal-derived factor-1. Blood 2002;99:2703-2711.

22 Salcedo R, Oppenheim JJ: Role of chemokines in angiogenesis: CXCL12/SDF-1 and CXCR4 interaction, a key regulator of endothelial cell responses. Microcirculation 2003; 10:359-370.

23 Peled A, Kollet O, Ponomaryov T, Petit I, Franitza S, Grabovsky V, et al: The chemokine SDF-1 activates the integrins LFA-1, VLA-4 and VLA -5 on immature human CD34+ cells: role in transendothelial/stromal migration and engraftment of NOD/SCID mice. Blood 2000;95:3289-3296.

24 Zemani F, Silvestre J-S, Fauvel-Lafeve F, Bruel A, Vilar J, Bieche I, et al: Ex vivo priming of endothelial progenitor cells with SDF-1 before transplantation could increase their proangiogenic potential. Arterioscler Thromb Vasc Biol 2008;28:644-650.

25 Silva EA, Kim E-S, Kong HJ, Mooney DJ: Material-based deployment enhances efficacy of endothelial progenitor cells. Proc Natl Acad Sci U S A 2008;105:14347-14352.
26 Yuen WW, Du NR, Shvartsman D, Arany PR, Lam H, Mooney DJ: Statistical platform to discern spatial and temporal coordination of endothelial sprouting. Integr Biol 2012;4: 292-300.

27 Anderson EM, Kwee BJ, Lewin SA, Raimondo T, Mehta M, Mooney DJ: Local delivery of VEGF and SDF enhances endothelial progenitor cell recruitment and resultant recovery from ischemia. Tissue Eng Part A 2015;21: 1217-1227.

28 Elmadbouh I, Haider HK, Jiang S, Idris NM, Lu G, Ashraf M: Ex vivo delivered stromal cell-derived factor-1alpha promotes stem cell homing and induces angiomyogenesis in the infarcted myocardium. J Mol Cell Cardiol 2007;42:792-803.

29 Isner JM: Tissue responses to ischemia: local and remote responses for preserving perfusion of ischemic muscle. J Clin Invest 2000; 106:615-619.

30 Salcedo R, Wasserman K, Young H a, Grimm MC, Howard OM, Anver MR, et al: Vascular endothelial growth factor and basic fibroblast growth factor induce expression of CXCR4 on human endothelial cells: in vivo neovascularization induced by stromal cell-derived factor-1alpha. Am J Pathol 1999;154:11251135.

-31 Wang S, Ma N, Kawanishi S, Hiraku Y, Oikawa S, Xie Y, et al: Relationships of alphaSMA-positive fibroblasts and SDF-1-positive tumor cells with neoangiogenesis in nasopharyngeal carcinoma. Biomed Res Int 2014; 2014:507353.

- 32 Ii M, Horii M, Yokoyama A, Shoji T, Mifune Y, Kawamoto A, et al: Synergistic effect of adipose-derived stem cell therapy and bone marrow progenitor recruitment in ischemic heart. Lab Invest 2011;91:539-552. 